Article

\title{
On the Isomorphism between Dissipative Systems, Fractal Self-Similarity and Electrodynamics. Toward an Integrated Vision of Nature
}

\section{Giuseppe Vitiello $^{1,2}$}

${ }^{1}$ Dipartimento di Fisica “E. R. Caianiello”, Università di Salerno, Fisciano (Salerno) 84084, Italy; E-Mail: vitiello@sa.infn.it

${ }^{2}$ INFN, Sezione di Napoli, Gruppo Collegato Salerno, Fisciano (Salerno) 84084, Italy

Received: 9 April 2014; in revised form: 5 May 2014 / Accepted: 12 May 2014 /

Published: 14 May 2014

\begin{abstract}
In electrodynamics there is a mutual exchange of energy and momentum between the matter field and the electromagnetic field and the total energy and momentum are conserved. For a constant magnetic field and harmonic scalar potential, electrodynamics is shown to be isomorph to a system of damped/amplified harmonic oscillators. These can be described by squeezed coherent states which in turn are isomorph to self-similar fractal structures. Under the said conditions of constant magnetic field and harmonic scalar potential, electrodynamics is thus isomorph to fractal self-similar structures and squeezed coherent states. At a quantum level, dissipation induces noncommutative geometry with the squeezing parameter playing a relevant role. Ubiquity of fractals in Nature and relevance of coherent states and electromagnetic interaction point to a unified, integrated vision of Nature.
\end{abstract}

Keywords: electrodynamics; fractal self-similarity; dissipation; coherence

\section{Introduction}

It is well known, and well established by experimental observations, that systems characterized by observable ordered symmetry patterns, such as crystal, ferromagnets, superconductors, may be described in quantum field theory (QFT) by coherent boson condensation in the ground state which arises as a consequence of the spontaneous breakdown of symmetry mechanism [1-3]. Even in high energy physics, such a mechanism, with the recent discovery of the Higgs particle, proves to be crucial and the vacuum state appears to have the structure of a coherent condensate. The study of topologically 
non-trivial "extended objects", such as vortices, crystal dislocations, domain walls and other soliton solutions, also shows that boson condensation is at the root of the formation of such macroscopic quantum systems, as also confirmed in many laboratory observations [4]. In recent works it has been shown that also the formalism describing fractal self-similar structures is isomorph to the one of coherent boson condensates, in particular to squeezed generalized $S U(1,1)$ coherent states [5-8]. These last ones, on the other hand, provide a representation of the system of damped/amplified oscillators, which is a prototype of dissipative system and the environment or bath in which it is embedded. In this paper, by resorting to the well known result in classical and quantum electrodynamics on the conservation of the energy-momentum tensor, the isomorphism is shown to exist, under convenient conditions, also between electrodynamics and the system of damped/amplified oscillators, and thus squeezed $S U(1,1)$ coherent states and fractal self-similar structures. Such a result, in view of the ubiquity of fractals in Nature and of the relevance of coherent states and electrodynamics, opens the way to an integrated vision of Nature. The plan of the paper is the following. In Section 2 the conservation of energy-momentum in electrodynamics is reviewed, also in connection with the system of damped/amplified oscillators, whose analysis is continued in Section 3 and its quantization formalism is presented in Section 4. Finally, coherence and fractal self-similarity is discussed in Section 5. Section 6 is devoted to conclusions and the emergence of an integrated vision of Nature is commented upon.

\section{The Conservation of Energy and Momentum in Electrodynamics}

In classical electrodynamics, as well as in quantum electrodynamics (QED), it is well known that the conservation of the electromagnetic (em) energy-momentum tensor $T^{\mu \nu}$ holds: $\partial_{\mu} T^{\mu \nu}=0$. The system of the matter field $\psi$ and of the em gauge field $A^{\mu}$ is a closed system, $\left\{\psi, A^{\mu}\right\}$ : there is no energy/momentum flowing out of it. However, one may easily realize that the conservation of $T^{\mu \nu}$ arises from the compensation of the variations of the matter part $T_{m}^{\mu \nu}$ and the em part $T_{\gamma}^{\mu \nu}$ of the total $T^{\mu \nu}$ : $\partial_{\mu} T_{m}^{\mu \nu}=-\partial_{\mu} T_{\gamma}^{\mu \nu}$, so that $\partial_{\mu} T^{\mu \nu}=\partial_{\mu}\left(T_{m}^{\mu \nu}+T_{\gamma}^{\mu \nu}\right)=0$. In particular one finds:

$$
\begin{gathered}
\partial_{\mu} T_{m}^{\mu \nu}=e F^{\alpha \nu} J_{\alpha} \\
\partial_{\mu} T_{\gamma}^{\mu \nu}=-e F^{\alpha \nu} J_{\alpha}
\end{gathered}
$$

where $J_{\alpha}$ denotes the current, $e$ is the charge and as usual $F^{\alpha \beta}=\partial^{\beta} A^{\alpha}-\partial^{\alpha} A^{\beta}\left(g^{\mu \nu}=(1,-1,-1,-1)\right.$, $\mu=0,1,2,3 ; \hbar=1=c$ will be used throughout the paper). It needs to be stressed that Equations (1) and (2) are characterizing equations for classical and quantum electrodynamics, they fully express the dynamical content of Maxwell equations and the associated conservation laws. We see that the non-vanishing divergences of $T_{m}^{\mu \nu}$ and $T_{\gamma}^{\mu \nu}$ compensate each other, which, stated in different words, means that the em field acts as the reservoir for the dissipative matter field system, or vice-versa, exchanging the roles of the matter field and the em field. Such a conclusion is a general one. For what concerns our discussion there is no need to specify the boson or fermion nature of $\psi(x)$. The Lagrangian is only required to be invariant under local gauge transformations. Recalling now that the energy-momentum vector $P^{\mu}$ is given by

$$
P^{\mu}=\int T^{\mu 0} d^{3} x, \quad \mu=0,1,2,3
$$


and $E^{i}=F^{0 i}, B^{i}=\epsilon_{i j k} F^{k j}, i, j, k=1,2,3$, with $E^{i}$ and $B^{i}$ denoting the $i$-component of the electric and magnetic fields, $\mathbf{E}$ and $\mathbf{B}$, respectively, volume integration of Equations (1) and (2) gives for $\nu=0$ the rate of changes in time of the energy of the matter field and em field, $\mathcal{E}_{m}$ and $\mathcal{E}_{\gamma}$, respectively:

$$
\partial_{0} \mathcal{E}_{m}=e \mathbf{E} \cdot \mathbf{v}=-\partial_{0} \mathcal{E}_{\gamma}
$$

For $\nu=i=1,2,3$, integration of Equations (1) and (2) over the volume gives

$$
\begin{gathered}
\partial_{0} P_{m}^{i}=e E^{i}+e(\mathbf{v} \times \mathbf{B})^{i} \\
\partial_{0} P_{\gamma}^{i}=-e E^{i}-e(\mathbf{v} \times \mathbf{B})^{i}
\end{gathered}
$$

namely, the Lorentz forces $\mathbf{F}_{m}$ and $\mathbf{F}_{\gamma}$, acting on two opposite charges with same velocity $\mathbf{v}$ in the same electric and magnetic fields. They are equal and opposite, component by component. We thus see that the energy-momentum vector is conserved only provided the matter field is considered together with the em field (and vice-versa). Each of the fields, separately considered, behaves as an open system. Only the system made of both fields is non-dissipative.

Suppose now that, at least in some space-time region, the magnetic field $\mathbf{B}$ be a constant vector, thus described by the vector potential

$$
\mathbf{A}=\frac{1}{2} \mathbf{B} \times \mathbf{r}
$$

where $\mathbf{r}=\left(x_{1}, x_{2}, x_{3}\right)$. It is $\mathbf{B}=\nabla \times \mathbf{A}, \nabla \cdot \mathbf{A}=0$ and, without loss of generality, choose the reference frame so that $\boldsymbol{B}=\boldsymbol{\nabla} \times \boldsymbol{A}=-B \hat{\mathbf{3}}$. Then, $A_{3}=0$ and by using $\epsilon_{12}=-\epsilon_{21}=1 ; \epsilon_{i i}=0$,

$$
A_{i}=\frac{B}{2} \epsilon_{i j} x_{j}, \quad i, j=1,2
$$

and the third component, $i=3$, of $(\mathbf{v} \times \mathbf{B})$ vanishes. Assume also that $\boldsymbol{E}$ is given by the gradient of the harmonic potential $\Phi \equiv \frac{k}{2 e}\left(x_{1}{ }^{2}-x_{2}{ }^{2}\right) \equiv \Phi_{1}-\Phi_{2}, \boldsymbol{E}=-\nabla \Phi$; and $E_{3}=0$. We may thus limit our analysis to the $i=1,2$ components in Equations (5) and (6). Then let $i=1$ in Equation (5) and put $B \equiv \gamma / e$. Use of Equation (8) gives

$$
m \ddot{x}_{1}+\gamma \dot{x}_{2}+k x_{1}=0
$$

where $m, \gamma$ and $k$ are time independent quantities and the first member of Equation (5) has been put equal to $m \ddot{x}_{1}$ (and is equal and opposite to the $i=1$ component of the force in Equation (6)). By considering $i=2$ in Equation (6) gives

$$
m \ddot{x}_{2}+\gamma \dot{x}_{1}+k x_{2}=0
$$

(note that similar conclusion may be reached considering $i=2$ in Equation (5) and $i=1$ in Equation (6)). In the case of constant magnetic field and harmonic scalar potential Equations (9) and (10) thus provide a representation of electrodynamics expressed by Equations (5) and (6).

The problem of the separation of the $x_{1}$ and $x_{2}$ variables is discussed in the following Section. Let me close the present one by observing that these equations can be derived from the Lagrangian

$$
L=\frac{m}{2}\left(\dot{x}_{1}^{2}-\dot{x}_{2}^{2}\right)+e\left(\dot{x}_{1} A_{1}+\dot{x}_{2} A_{2}\right)-e \Phi
$$


which shows that Equations (9) and (10) describe indeed opposite charges in a constant magnetic field and a harmonic scalar potential and exhibits the correct coupling between the current and the vector potential field (charge density is $e \rho$ and $e \mathbf{J}=e \rho \mathbf{v}$, with $\mathbf{v}=d \mathbf{r} / d t$ the velocity). Equation (11) can be written also as

$$
L=\frac{1}{2 m}\left(m \dot{x}_{1}+e_{1} A_{1}\right)^{2}-\frac{1}{2 m}\left(m \dot{x}_{2}+e_{2} A_{2}\right)^{2}-\frac{e^{2}}{2 m}\left(A_{1}{ }^{2}-A_{2}^{2}\right)-e \Phi
$$

That $x_{2}$ behaves as the em field for $x_{1}$, and vice-versa is explicitly shown by considering the Hamiltonian $[9,10]$

$$
H=H_{1}-H_{2}=\frac{1}{2 m}\left(p_{1}-e_{1} A_{1}\right)^{2}+e_{1} \Phi_{1}-\frac{1}{2 m}\left(p_{2}+e_{2} A_{2}\right)^{2}+e_{2} \Phi_{2}
$$

We see that in the least energy state (where $H=0, H_{1}=H_{2}$ ) the respective contributions to the energy compensate each other. Equations (9) and (10) are derived from Equation (12) in the usual form

$$
\frac{d}{d t}\left(m \dot{x}_{i}+e_{i} A_{i}\right)=-e \partial_{i} \Phi_{i}+e_{i} \partial_{i} v_{j} A_{j}
$$

where $i, j=1,2, i \neq j$, no sum on $i, j, \Phi_{i} \equiv \frac{k}{2 e} x_{i}{ }^{2}, e_{1}=e=-e_{2}, \partial_{i} \equiv \frac{\partial}{\partial x_{i}}$ and it is $\frac{d}{d t} A_{i}=v_{j} \partial_{j} A_{i}$. In summary, one of the oscillators may be considered to represent the em field in which the other one is embedded.

\section{The Damped Oscillator and Its Double}

In order to separate $x_{1}$ and $x_{2}$ variables one may use the canonical transformations

$$
x(t)=\frac{x_{1}(t)+x_{2}(t)}{\sqrt{2}}, y(t)=\frac{x_{1}(t)-x_{2}(t)}{\sqrt{2}}
$$

thus obtaining from Equations (9) and (10) the couple of damped and amplified harmonic oscillators (dho):

$$
\begin{aligned}
& m \ddot{x}+\gamma \dot{x}+k x=0 \\
& m \ddot{y}-\gamma \dot{y}+k y=0
\end{aligned}
$$

respectively. The $y$ oscillator may be considered as the reservoir (or the environment, or the bath) for the $x$ oscillator (or vice-versa), which is consistent with the remark made above on the mutual exchange of energy-momentum between the em field and the matter field. In other words, the $y$ oscillator acts as the sink into which the energy dissipated by the $x$ oscillator flows. The couple of the $x$ oscillator and its "double" oscillator $y$ form the closed system $(x-y)$. Moreover, Equation (17) is the time-reversed image $(\gamma \rightarrow-\gamma)$ of Equation (16) (or vice-versa).

By introducing the pseudo-euclidean metric

$$
r(t)^{2} \equiv x_{1}(t)^{2}-x_{2}(t)^{2}
$$

namely

$$
x(t)=\frac{1}{\sqrt{2}} r(t) e^{u(t)}, y(t)=\frac{1}{\sqrt{2}} r(t) e^{-u(t)}
$$


Equations (16) and (17) are found to be formally equivalent to the equation for the harmonic oscillator $r(t)$ representing the global $(x-y)$-system:

$$
m \ddot{r}+K r=0
$$

where $K \equiv \frac{1}{m}\left(k-\frac{\gamma^{2}}{4 m}\right) \equiv m \Omega^{2}$, assuming $k>\frac{\gamma^{2}}{4 m}$, provided $u(t) \equiv-\frac{\gamma}{2 m} t$, consistently with the time independence of the coefficients $m, \gamma$ and $k$. The reverse is also true: the oscillator (20) is decomposed into two damped/amplified oscillators (16) and (17) when the pseudo-euclidean metric is adopted [9,10]. On the contrary, the $r$-oscillator is decomposed into two undamped oscillators when the euclidean metric, $r^{2} \equiv x_{1}^{2}+x_{2}^{2}, x_{1}=r \cos \alpha, x_{2}=r \sin \alpha$, is used.

The Lagrangian (11) and the Hamiltonian (13), rewritten in terms of the $(x-y)$-system, become

$$
\begin{gathered}
L=m \dot{x} \dot{y}+\frac{\gamma}{2}(x \dot{y}-\dot{x} y)-k x y \\
H=\frac{1}{m} p_{x} p_{y}+\frac{1}{2 m} \gamma\left(y p_{y}-x p_{x}\right)+\left(k-\frac{\gamma^{2}}{4 m}\right) x y
\end{gathered}
$$

respectively, with conjugate momenta $p_{x}=m \dot{y}-\frac{\gamma}{2} y$, and $p_{y}=m \dot{x}+\frac{\gamma}{2} x$ and the common frequency of the two oscillators $\Omega \equiv\left[\frac{1}{m}\left(k-\frac{\gamma^{2}}{4 m}\right)\right]^{\frac{1}{2}}, k>\frac{\gamma^{2}}{4 m}$ (i.e., assuming no overdamping). Note that conjugate momenta cannot be defined without introducing the doubled mode $y$.

Summing up, the system made of two separate modes describing different physical evolutions (damping and amplification) is a closed system. Considering only one of them breaks the time-reversal symmetry and a partition on the time axis is induced, implying that positive and negative time directions are associated with the two separate modes. The canonical formalism is not able to describe dissipative systems, i.e., cannot describe separately each one of the modes. It can only describe the closed system. Remarkably, the two separate non-conserving modes Equations (5) and (6) (and Equation (4)) out of which electrodynamics is made are associated with charge conjugation $(e \leftrightarrow-e)$.

The issue of the quantization of the system of Equations (16) and (17) will be discussed in the following Section (see [11-13]).

\section{Dissipation and Quantization}

The issue of quantum dissipation in connection with the damped/amplified system Equations (16) and (17) has been considered in details in literature [3,11-13]). Therefore here only the resulting formulas which are relevant to our discussion will be reported.

Canonical quantization of the oscillator system Equations (16) and (17) is performed as customary by introducing the commutators $\left[x, p_{x}\right]=i \hbar=\left[y, p_{y}\right],[x, y]=0=\left[p_{x}, p_{y}\right]$ and the annihilation and creation operators

$$
\begin{aligned}
a & \equiv\left(\frac{1}{2 \hbar \Omega}\right)^{\frac{1}{2}}\left(\frac{p_{x}}{\sqrt{m}}-i \sqrt{m} \Omega x\right) ; a^{\dagger} \equiv\left(\frac{1}{2 \hbar \Omega}\right)^{\frac{1}{2}}\left(\frac{p_{x}}{\sqrt{m}}+i \sqrt{m} \Omega x\right) \\
b & \equiv\left(\frac{1}{2 \hbar \Omega}\right)^{\frac{1}{2}}\left(\frac{p_{y}}{\sqrt{m}}-i \sqrt{m} \Omega y\right) ; b^{\dagger} \equiv\left(\frac{1}{2 \hbar \Omega}\right)^{\frac{1}{2}}\left(\frac{p_{y}}{\sqrt{m}}+i \sqrt{m} \Omega y\right)
\end{aligned}
$$


with $\left[a, a^{\dagger}\right]=1=\left[b, b^{\dagger}\right], \quad[a, b]=0=\left[a, b^{\dagger}\right]$. By use of the canonical linear transformations $A \equiv \frac{1}{\sqrt{2}}(a+b), B \equiv \frac{1}{\sqrt{2}}(a-b)$ and putting $\Gamma \equiv \frac{\gamma}{2 m}$, the Hamiltonian $H$ is obtained [11]

$$
\begin{gathered}
H=H_{0}+H_{I} \\
H_{0}=\hbar \Omega\left(A^{\dagger} A-B^{\dagger} B\right), H_{I}=i \hbar \Gamma\left(A^{\dagger} B^{\dagger}-A B\right)
\end{gathered}
$$

By defining $J_{+}=A^{\dagger} B^{\dagger}, \quad J_{-}=J_{+}^{\dagger}=A B, \quad J_{3}=\frac{1}{2}\left(A^{\dagger} A+B^{\dagger} B+1\right),\left[J_{+}, J_{-}\right]=-2 J_{3}$, $\left[J_{3}, J_{ \pm}\right]= \pm J_{ \pm}$the two mode realization of the algebra $\operatorname{su}(1,1)$ is obtained. The $S U(1,1)$ Casimir operator $\mathcal{C}$ is given by $\mathcal{C}^{2}=\frac{1}{4}\left(A^{\dagger} A-B^{\dagger} B\right)^{2}$, so that $\left[H_{0}, H_{I}\right]=0$. The initial condition of positiveness for the eigenvalues of $H_{0}$ are thus protected against the danger of transitions to negative energy states.

The vacuum state is $|0\rangle \equiv\left|n_{A}=0, n_{B}=0\right\rangle=|0\rangle \otimes|0\rangle$, with $n_{A}$ and $n_{B}$ the number of $A$ and $B$ 's and $(A \otimes 1)|0\rangle \otimes|0\rangle \equiv A|0\rangle=0 ;(1 \otimes B)|0\rangle \otimes|0\rangle \equiv B|0\rangle=0$. Its time evolution is controlled by $H_{I}$ and given by $|0(t)\rangle=e^{-i t \frac{H}{\hbar}}|0\rangle=e^{-i t \frac{H_{I}}{\hbar}}|0\rangle$, with $\langle 0(t) \mid 0(t)\rangle=1, \forall t$, and

$$
\lim _{t \rightarrow \infty}\langle 0(t) \mid 0\rangle \propto \lim _{t \rightarrow \infty} \exp (-t \Gamma)=0
$$

One finds that states generated by $B^{\dagger}$ represent the sink where the energy dissipated by the quantum damped oscillator flows: the $B$-oscillator represents the reservoir or heat bath coupled to the $A$-oscillator [11]. The instability (decay) of the vacuum is expressed by Equation (27), which shows that time evolution leads out of the Hilbert space of the states. Therefore, the framework of quantum mechanics is not suitable for the canonical quantization of the damped harmonic oscillator. The proper framework is the one of QFT [11], where the time evolution operator $\mathcal{U}(t)$ and the vacuum are formally (at finite volume) given by

$$
\begin{aligned}
\mathcal{U}(t) & =\prod_{\kappa} \exp \left(\Gamma_{\kappa} t\left(A_{\kappa}^{\dagger} B_{\kappa}^{\dagger}-A_{\kappa} B_{\kappa}\right)\right) \\
|0(t)\rangle & =\prod_{\kappa} \frac{1}{\cosh \left(\Gamma_{\kappa} t\right)} \exp \left(\tanh \left(\Gamma_{\kappa} t\right) A_{\kappa}^{\dagger} B_{\kappa}^{\dagger}\right)|0\rangle
\end{aligned}
$$

respectively, with $\langle 0(t) \mid 0(t)\rangle=1, \forall t$. In the infinite volume limit we have (for $\int d^{3} \kappa \Gamma_{\kappa}$ finite and positive)

$$
\langle 0(t) \mid 0\rangle \rightarrow 0 \text { as } V \rightarrow \infty \forall t
$$

Here the relation $\sum_{\kappa} \rightarrow \frac{V}{(2 \pi)^{3}} \int d^{3} \kappa$ has been used. Note that $\left\langle 0(t) \mid 0\left(t^{\prime}\right)\right\rangle \rightarrow 0$ as $V \rightarrow \infty \forall t$ and $t^{\prime}, t^{\prime} \neq t$ : a representation $\{|0(t)\rangle\}$ of the canonical commutation relations (CCR) is defined at each time $t$ and is unitarily inequivalent to any other representation $\left\{\left|0\left(t^{\prime}\right)\right\rangle, \forall t^{\prime} \neq t\right\}$ in the infinite volume limit. The system thus evolves in time through unitarily inequivalent representations of CCR [11]. Note that $|0(t)\rangle$ is a two-mode time dependent generalized $S U(1,1)$ coherent state $[3,11,14,15]$ where $A$ and $B$ are entangled modes, which is consistent with the entanglement between the (charged) matter field and the em field in QED. Remarkably, in QFT the entanglement notion enters in a natural way through the coherent state structure of the vacuum state (in the present case the coherent $S U(1,1)$ boson condensation of the couple $A B$ in $|0(t)\rangle$ ). Moreover, entanglement cannot be destroyed by the action of any unitary operator since it characterizes unitarily inequivalent representations, a feature absent in quantum mechanics. 
The number of $A_{\kappa}$ (or $B_{\kappa}$ ) modes in $|0(t)\rangle$ is given by

$$
\mathcal{N}_{A_{\kappa}}(t)=\left\langle 0(t)\left|A_{\kappa}^{\dagger} A_{\kappa}\right| 0(t)\right\rangle=\sinh ^{2} \Gamma_{\kappa} t
$$

$|0(t)\rangle$ turns out to be a squeezed coherent state characterized by the $q$-deformation of Lie-Hopf algebra [3,16-18] and provides a representation of the CCR at finite temperature which is equivalent [11] to the Thermo Field Dynamics representation $\{|0(\beta)\rangle\}[1,2]$.

Time evolution is controlled by the entropy variations [11,19], which is consistent with the fact that dissipation implies breaking of time-reversal invariance (i.e., the choice of a privileged direction in time evolution, the arrow of time). Heat dissipation $d Q=\frac{1}{\beta} d S$ is given by the variations in time of the number of particles condensed in the vacuum.

The classical system of oscillators considered above is known to belong to the class of deterministic systems à la 't Hooft [20-24] (those systems that remain deterministic even when described by means of Hilbert space techniques). The quantum harmonic oscillator emerges from the classical (dissipative) system when one imposes a constraint on the Hilbert space of the form $J_{2}|0\rangle=0$. For further details on 't Hooft analysis see [20-24].

In conclusion, an isomorphism has been established between electrodynamics and the $S U(1,1)$ couple of damped/amplified quantum oscillators. It is interesting that according to a recent result [25] the quantized electromagnetic field appears to be naturally described by $s u(1,1)$ rather than Weyl-Heisenberg algebra.

In the following Section, our discussion goes further by showing the isomorphism between the generalized coherent states entering the quantum dissipation formalism and self-similarity properties of fractal structures. On the basis of the discussion presented above, the isomorphism between fractal self-similarity and QED is also implied.

\section{Coherence and Fractal Self-Similarity}

In the previous Sections, the system of damped/amplified harmonic oscillators Equations (16) and (17) has been shown to be isomorph to QED under proper conditions (i.e., for a constant magnetic field and for a harmonic scalar potential): one of the oscillator may be considered to represent the em field in which the other one is embedded. In this Section, by resorting to the results of [5-8], we will show that an isomorphism exists between the fractal self-similarity properties and the coherent state structure in QFT. In view of the isomorphism discussed in the previous Sections, this also establishes a relation between electrodynamics and fractal self-similarity.

I will consider the case of the logarithmic spiral and the Koch curve. The conclusions can be extended to other examples of deterministic fractals (namely those which are generated iteratively according to a prescribed recipe), such as the Sierpinski gasket and carpet, the Cantor set, etc. [26,27].

Let me start with the logarithmic spiral (Figure 1). Its representation in polar coordinates $(r, \theta)$ is $[27,28]$ :

$$
r=r_{0} e^{d \theta}
$$


with arbitrary real constants $r_{0}$ and $d$ and $r_{0}>0$. Equation (32) is represented by the straight line of slope $d$ in a $\log -\log$ plot with abscissa $\theta=\ln e^{\theta}$ :

$$
d \theta=\ln \frac{r}{r_{0}}
$$

Figure 1. The anti-clockwise and the clockwise logarithmic spiral.

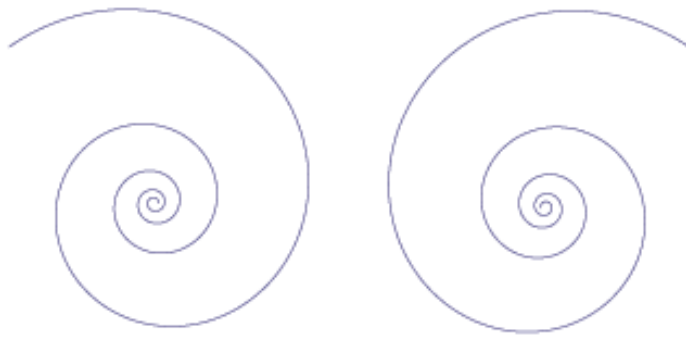

The self-similarity property is represented by the constancy of the angular coefficient $\tan ^{-1} d$. Rescaling $\theta \rightarrow n \theta$ affects $r / r_{0}$ by the power $\left(r / r_{0}\right)^{n}$. The parametric equations of the spiral are:

$$
\begin{aligned}
& \xi=r(\theta) \cos \theta=r_{0} e^{d \theta} \cos \theta \\
& \eta=r(\theta) \sin \theta=r_{0} e^{d \theta} \sin \theta
\end{aligned}
$$

The point $z=\xi+i \eta=r_{0} e^{d \theta} e^{i \theta}$ on the spiral in the complex $z$-plane is fully specified once the the sign of $d \theta$ is assigned. The completeness of the (hyperbolic) basis $\left\{e^{-d \theta}, e^{+d \theta}\right\}$ requires that the points $z_{1}=r_{0} e^{-d \theta} e^{-i \theta}$ and $z_{2}=r_{0} e^{+d \theta} e^{+i \theta}$ need both to be considered. Opposite signs for the imaginary exponent $\pm i \theta$ also have been considered for convenience. By using the parametrization $\theta=\theta(t), z_{1}$ and $z_{2}$ can be shown to solve the equations ("dot" denotes derivative with respect to $t$ )

$$
\begin{aligned}
& m \ddot{z}_{1}+\gamma \dot{z}_{1}+\kappa z_{1}=0 \\
& m \ddot{z}_{2}-\gamma \dot{z}_{2}+\kappa z_{2}=0
\end{aligned}
$$

respectively, provided that

$$
\theta(t)=\frac{\gamma}{2 m d} t=\frac{\Gamma}{d} t
$$

up to an arbitrary additive constant; $m, \gamma$ and $\kappa$ are positive real constants. Thus the logarithmic spirals are described by $z_{1}(t)=r_{0} e^{-i \Omega t} e^{-\Gamma t}$ and $z_{2}(t)=r_{0} e^{+i \Omega t} e^{+\Gamma t}$ solutions of Equations (36) and (37) and the parameter $t$ can be interpreted as the time parameter. The notations $\Gamma \equiv \gamma / 2 m$ and $\Omega^{2}=(1 / m)\left(\kappa-\gamma^{2} / 4 m\right)=\Gamma^{2} / d^{2}$, with $\kappa>\gamma^{2} / 4 m$, are the same as in Section 3. Note that by putting $\left[z_{1}(t)+z_{2}^{*}(-t)\right] / 2=x(t)$ and $\left[z_{1}^{*}(-t)+z_{2}(t)\right] / 2=y(t)$, Equations (36) and (37) reduce to Equations (16) and (17) (namely they provide an equivalent representation of Equations (5) and (6)). Observe that $\theta(T)=2 \pi$ at $T=2 \pi d / \Gamma$. At $t=m T, z_{1}=r_{0}\left(e^{-2 \pi d}\right)^{m}, z_{2}=r_{0}\left(e^{2 \pi d}\right)^{m}$, with integer $m=1,2,3 \ldots$ We thus see that the continuous character of Equations (34) and (35) (which is reflected in the continuous time evolution according to Equation (38)) also includes as a subset, due to the T integer multiplicity, the discrete group transformation $z_{1}(m)=r_{0}\left(e^{-2 \pi d}\right)^{m} \rightarrow z_{1}(m+1)=r_{0}\left(e^{-2 \pi d}\right)^{(m+1)}=$ $z_{1}(m)\left(e^{-2 \pi d}\right)$. This suggests to us that the isomorphism we refer to appears as a homomorphism. Such an occurrence is indeed implicit in the very same structure of Equations (34) and (35) describing the 
logarithmic spiral. A similar situation occurs in the case of the Koch curve and other fractals considered below. We plan to present a deeper analysis of this point in a future paper. The spiral "angular velocity" is $|d \theta / d t|=|\Gamma / d|$. Our discussion also includes the golden spiral and its relation with Fibonacci progression (see the Appendix).

By proceeding in a similar way as done in previous Sections, the Lagrangian, the Hamiltonian Equations (25) and (26) and also the other formulas for the evolution operator, the ground state, etc., Equations (28)-(31) are obtained for the spiral when working in the proper QFT frame. The breakdown of time-reversal symmetry is again associated with the choice of a privileged direction in time evolution and the entropy operator $S$ may be defined. The left-handed chirality spiral (direct spiral, $q \equiv e^{-d \theta}>1$ ) is the time-reversed, but distinct, image of the right-handed chirality spiral (indirect spiral, $q<1$ ).

The Hamiltonian $H$ is actually the fractal free energy for the coherent boson condensation process out of which the fractal is formed. $H_{0}=2 \hbar \Omega \mathcal{C}$ can be identified with the "internal energy" $U$ and $2 J_{2}$ with the entropy $S$. From Equation (26) and the defining equation for the temperature $T$ (putting $k_{B}=1$ ), we have $\partial S / \partial U=1 / T$ and obtain $T=\hbar \Gamma$. Thus, $H$ represents the free energy $\mathcal{F}=U-T S$. The heat contribution in $\mathcal{F}$ is given by $2 \Gamma J_{2}$ and $\left.(\partial \mathcal{F} / \partial T)\right|_{\Omega}=-2 J_{2}$. The temperature $T=\hbar \Gamma$ is found to be proportional to the background zero point energy: $\hbar \Gamma \propto \hbar \Omega / 2$ [3,22-24].

The evolution operator $\mathcal{U}(t)$, when written in terms of the $a$ and $b$ operators (see Section 4), becomes [3,16-18]

$$
\mathcal{U}(t)=\exp \left(-\frac{\Gamma t}{2}\left(\left(a^{2}-a^{\dagger 2}\right)-\left(b^{2}-b^{\dagger 2}\right)\right)\right)
$$

and it is recognized to be the two mode squeezing generator with squeezing parameter $\zeta=-\Gamma t$. The $S U(1,1)$ generalized coherent state (29) is thus a squeezed state.

Consider now the examples of the Koch curve [26,27]. Let $u_{0}=1$ for the initial stage $u_{0}$ (see Figure 2) [5]. The $n$-th step or stage is denoted by $u_{n, q}(\alpha)$, with $\alpha=4$ and $q=1 / 3^{d}[6,7]$. One has

$$
u_{n, q}(\alpha)=(q \alpha)^{n}=1, \quad \text { for any } n
$$

which gives the self-similarity, or fractal dimension [27] $d=\ln 4 / \ln 3 \approx 1.2619$. It is important to stress that self-similarity is properly defined only in the $n \rightarrow \infty$ limit. Put $q=e^{-d \theta}$ and write the self-similarity equation for $n=1$ in polar coordinates as $u=u_{0} \alpha e^{d \theta}$, which is similar to Equation (32) (or as $d \theta=\ln \alpha$ which is similar to Equation (33)). One can proceed then as in the case of the logarithmic spiral, the parametric equations for the fractal in the $z$-plane can be written, and so on to obtain the fractal Hamiltonian and free energy. The exponential operator in the evolution operator $\mathcal{U}(t)$ is

$$
\left(\left(c^{2}-c^{\dagger 2}\right)-\left(\tilde{c}^{2}-\tilde{c}^{\dagger 2}\right)\right)=-2\left(C^{\dagger} D^{\dagger}-C D\right)
$$

where $\tilde{c}$ and $\tilde{c}^{\dagger}$ denote the doubled degrees of freedom and $C \equiv \frac{1}{\sqrt{2}}(c+\tilde{c}), D \equiv \frac{1}{\sqrt{2}}(c-\tilde{c})$.

In conclusion, the relation between the self-similarity of logarithmic spiral and the Koch curve and the $\mathrm{SU}(1,1)$ generalized coherent state is thus established.

It is also interesting to consider the relation of the Koch curve with Glauber coherent states. Indeed, apart the normalization factor $1 / \sqrt{n !}$, the functions $u_{n, q}(\alpha)$ are recognized to be the restriction to real $q \alpha$ of the functions

$$
u_{n, q}(\alpha)=\frac{(q \alpha)^{n}}{\sqrt{n !}}, \quad n \in \mathcal{N}_{+}, \quad q \alpha \in \mathcal{C}
$$


which form a basis in the space $\mathcal{F}$ of the entire analytic functions. Therefore, the fractal properties can be studied in $\mathcal{F}$, by restricting, at the end, the results to real $q \alpha, q \alpha \rightarrow \operatorname{Re}(q \alpha)$ [6,7]. The relation with coherent states is established by realizing that $\mathcal{F}$ provides the Fock-Bargmann representation of the Weyl-Heisenberg algebra [14], namely, the frame where (Glauber) coherent states are described. Define $q \equiv e^{\zeta}, \zeta \in \mathcal{C}$ and apply $q^{N}$ to the coherent state $|\alpha\rangle, N \equiv \alpha d / d \alpha$; one finds the so-called $q$-deformed coherent state

$$
q^{N}|\alpha\rangle=|q \alpha\rangle=\exp \left(-\frac{|q \alpha|^{2}}{2}\right) \sum_{n=0}^{\infty} \frac{(q \alpha)^{n}}{\sqrt{n !}}|n\rangle
$$

Figure 2. The first five stages of Koch curve.

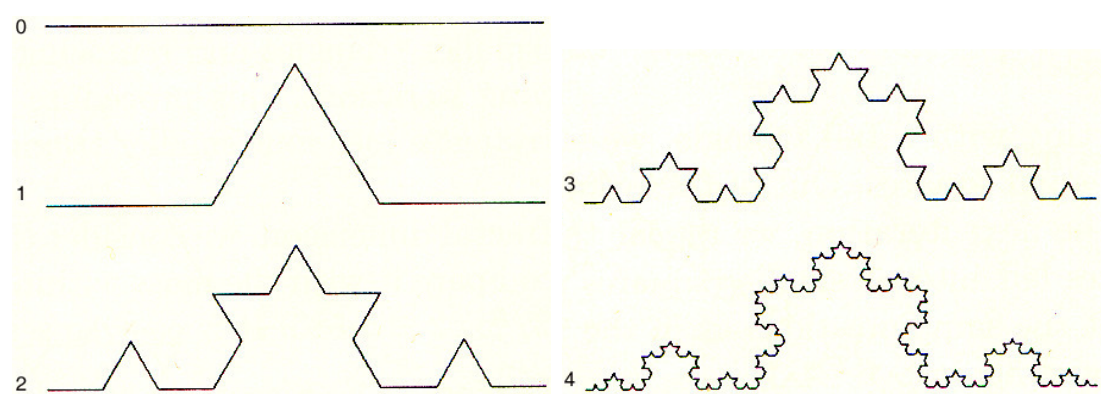

Here $a|\alpha\rangle=\alpha|\alpha\rangle$, with $a$ the annihilator operator. Apply $(a)^{n}$ to $|q \alpha\rangle$ and restrict then to real $q \alpha$ :

$$
\left\langle q \alpha\left|(a)^{n}\right| q \alpha\right\rangle=(q \alpha)^{n}=u_{n, q}(\alpha), \quad q \alpha \rightarrow \operatorname{Re}(q \alpha)
$$

which gives the $n$-th iteration stage of the fractal. The operator $(a)^{n}$ acts as a "magnifying" lens $[6,7,26]$. In conclusion, the one-to-one correspondence is established between the fractal $n$-th stage of iteration, $n=0,1,2, . ., \infty$, and the $n$-th term in the $q$-deformed coherent state Equation (43). $|q \alpha\rangle$ is a squeezed coherent state [16-18,29] with squeezing parameter $\zeta=\ln q$ and $q^{N}$, called the fractal operator [6,7], is the squeezing operator in $\mathcal{F}$. The squeezing transformation of the coherent state therefore describes the self-similarity properties of the Koch curve (and other fractals).

Let me close this Section with a comment on noncommutative geometry arising as an effect of dissipation [30]. In the $\left(z_{1}, z_{2}\right)$ plane use the notation $+\equiv 1$ and $-\equiv 2$, so that $p_{z_{ \pm}}$denote the momenta and $v_{ \pm}=\dot{z}_{ \pm}$the forward in time and backward in time velocities:

$$
v_{ \pm}=\frac{1}{m}\left(p_{z_{\mp}} \mp \frac{1}{2} \gamma z_{ \pm}\right), \quad \text { with } \quad\left[v_{+}, v_{-}\right]=-i \frac{\gamma}{m^{2}}
$$

The relation between dissipation and noncommutative geometry in the plane is thus obtained since the canonical set of conjugate position coordinates $\left(\xi_{+}, \xi_{-}\right)$can be then defined by putting $\xi_{ \pm}=\mp(\mathrm{m} / \gamma) v_{ \pm}$, with

$$
\left[\xi_{+}, \xi_{-}\right]=i \frac{1}{\gamma}
$$

The area $\mathcal{S}$ enclosed in the two paths $\mathcal{P}_{1}$ and $\mathcal{P}_{2}$ is proportional to the quantum dissipative interference phase $\vartheta=\mathcal{S} \gamma$ in the noncommutative plane, provided $z_{+} \neq z_{-}$.

On the other hand, the algebraic structure of the doubling of the degrees of freedom introduced above in order to close the system is a noncommutative one. The algebra is indeed duplicated by the map $\mathcal{A} \rightarrow \mathcal{A}_{1} \otimes \mathcal{A}_{2}$, which is the Hopf coproduct map $\mathcal{A} \rightarrow \mathcal{A} \otimes \mathbf{1}+\mathbf{1} \otimes \mathcal{A}$. Convenient combinations of 
the deformed coproducts in the $q$-deformed Hopf algebra, which are noncommutative [3,16-18,31,32], produce the Bogoliubov transformations of "angle" $\Gamma t$ and the $q$-deformation parameter controls the coherent condensate of the state $|0(t)\rangle$. This provides the physical meaning of the deformed Hopf algebraic structure and of the non-trivial topology of paths in the phase space $[31,33]$.

\section{Conclusions}

In this paper I have shown that, in space-time regions where the magnetic field may be approximated to be constant and the electric field is derivable from a harmonic potential, an isomorphism exists between (classical and quantum) electrodynamics and the set of damped and amplified oscillators representing a prototype of a dissipative system and the environment or bath in which it is embedded, respectively. It is known [5-8], on the other hand, that such a system of oscillators is isomorph to fractal self-similar structures. On the basis of these isomorphisms a link therefore is established between electrodynamics, dissipation and self-similarity. Moreover, the ground state of the oscillator system turns out to be a generalized $q$-deformed (or squeezed) $S U(1,1)$ coherent state. Coherent states thus appear to play a crucial role in our discussion. I also observe that quantum dissipation implies in a natural way noncommutative geometry in the plane and the notion of dissipative interference phase [6-8]. A rich, many-facet scenario thus emerges which certainly deserves further study. For example, it is interesting to observe that recently quantum electrodynamics has been shown [25] to be naturally described by $s u(1,1)$ rather than Weyl-Heisenberg algebra, which seems to agree with the general picture of the present paper establishing the relation between the $S U(1,1)$ system of damped/amplified oscillators and electrodynamics.

One more aspect which is related with the discussion here presented concerns with the description of fractal-like structures with self-similarity properties in terms of non-homogeneous Bose condensation. Indeed, in the present scheme they appear to be generated by coherent $S U(1,1)$ quantum condensation processes at the microscopic level, similar to "extended objects" or macroscopic quantum systems [1-4], such as crystals, ferromagnets and like systems in condensed matter physics characterized by ordered patterns. The macroscopic appearances (forms) of the fractals seems to emerge out of a process of morphogenesis as the macroscopic manifestation of the underlying dissipative, coherent quantum dynamics at the elementary level. An integrated vision of Nature resting, in its essence, on the paradigm of coherence and dissipation thus emerges. Even the sector of high energy physics, with the recent discovery of the Higgs boson and the coherent condensate structure of the vacuum, belongs to such a picture. Nature appears to be modulated by coherence, rather than being hierarchically layered in isolated compartments, in multi-coded collections of isolated systems and phenomena [5,34]. One might express this by saying that the dynamics of coherence is the primordial origin of codes. In this way codes are promoted from the (syntactic) level of pure information (à la Shannon) to the (semantic) level of meanings, expressions of coherent dynamical processes.

Let me close with a further comment concerning living matter physics. That coherence comes before the code seems to be supported also by the DNA duplication processes currently done in biological laboratories. In these polymerase chain reaction (PCR) processes, samples of water solutions containing the parent DNA (the template), primers, nucleotides and enzymes are submitted to sequences of thermal 
cycles. The DNA template transfers to the water in which it is embedded, by means of the emission of its em signal, the coherent structure of which its code is the expression. It turns out indeed that such an em signal has fractal-like self-similar structure and therefore, according to the theorem above discussed, it is expression of coherent dynamics. The duplicated DNA (reproducing the specific sequence of nucleotides of the parent DNA macromolecule), i.e., the genetic DNA code, is "re-constructed" as the dynamical output of the interaction of the coherent em signal with the water medium, and, through this, with the primers and nucleotides in the solution. In recent experiments [35] it has been possible to record the em signal emitted by aqueous solutions of DNA of viruses and bacteria and it has been shown that they have fractal-like self-similar structure. These signals have been used to irradiate (to "signalize") water. Then the original (parent) DNA has been reconstructed by use of usual PCR protocols, provided that primers and nucleotides, but not the template DNA, are added in the water solution. The occurrence of such a phenomenon also suggests that deformation (squeezing) of the signal coherence may induce dynamical epigenetic modifications, which may then signal the appearance of new meanings associated to deformed coherent signals. The DNA genetic code appears in conclusion to be the output of the coherent dynamics. In this way, it is subtracted from its purely phenomenological characterization, which is sometimes at the origin of dogmatic or even miraculous beliefs. In this view, DNA appears to be the vehicle through which the laws of form express themselves in living systems and coherence and its deformations propagate through duplication and multiplication processes.

\section{Conflicts of Interest}

The author declares no conflict of interest.

\section{References}

1. Umezawa, H.; Matsumoto, H.; Tachiki, M. Thermo Field Dynamics and Condensed States; North-Holland Publ. Co.: Amsterdam, The Netherlands, 1982.

2. Umezawa, H. Advanced Field Theory: Micro, Macro and Thermal Concepts; American Institute of Physics: Melville, NY, USA, 1993.

3. Blasone, M.; Jizba, P.; Vitiello, G. Quantum Field Theory and Its Macroscopic Manifestations; Imperial College Press: London, UK, 2011.

4. Bunkov, Y.M.; Godfrin, H. Topological Defects and the Nonequilibrium Dynamics of Symmetry Breaking Phase Transitions; Kluwer Academic Publishers: Dordrecht, The Netherlands, 2000.

5. Vitiello, G. Fractals, coherent states and self-similarity induced noncommutative geometry. Phys. Lett. 2012, A376, 2527-2532.

6. Vitiello, G. Coherent states, fractals and brain waves. New Math. Nat. Comput. 2009, 5, 245-264.

7. Vitiello, G. Fractals and the Fock-Bargmann representation of coherent states. In Quantum Interaction; Lecture Notes in Artificial Intelligence; Lawless, W., van Rijsbergen, K., Klusch, M., Eds.; Springer-Verlag: Berlin/Heidelberg, Germany, 2009; pp. 6-16.

8. Vitiello, G. Topological defects, fractals and the structure of quantum field theory. In Vision of Oneness; Licata, I., Sakaji, A.J., Eds.; Aracne Edizioni: Roma, Italy, 2011; pp. 155-179. 
9. Celeghini, E.; Graziano, E.; Nakamura, K.; Vitiello, G. Finite temperature quantum field theory and gauge field. Phys. Lett. 1992, B285, 98-102.

10. Blasone, M.; Graziano, E.; Pashaev, O.K.; Vitiello, G. Dissipation and topologically massive gauge theories in the pseudo-euclidean plane. Ann. Phys. 1996, 252, 115-132.

11. Celeghini, E.; Rasetti, M.; Vitiello, G. Quantum dissipation. Ann. Phys. 1992, 215, 156-170.

12. Blasone, M.; Srivastava, Y.N.; Vitiello, G.; Widom, A. Phase coherence in quantum brownian motion. Ann. Phys. 1998, 267, 61-74.

13. Srivastava, Y.N.; Vitiello, G.; Widom, A. Quantum dissipation and quantum noise. Ann. Phys. 1995, 238, 200-207.

14. Perelomov, A. Generalized Coherent States and Their Applications; Springer-Verlag: Berlin, Germany, 1986.

15. Klauder, J.R.; Skagerstam, B. Coherent States; World Scientific: Singapore, 1985.

16. Celeghini, E.; De Martino, S.; De Siena, S.; Rasetti, M.; Vitiello, G. Quantum groups, coherent states, squeezing and lattice quantum mechanics. Ann. Phys. 1995, 241, 50-67.

17. Celeghini, E.; Rasetti, M.; Tarlini, M.; Vitiello, G. $S U(1,1)$ squeezed states as damped oscillators. Mod. Phys. Lett. 1989, B3, 1213-1220.

18. Celeghini, E.; Rasetti, M.; Vitiello, G. On squeezing and quantum groups. Phys. Rev. Lett. 1991, 66, 2056-2059.

19. De Filippo, S.; Vitiello, G. Vacuum structure for unstable particles. Lett. Nuovo Cim. 1977, 19, 92-96.

20. 't Hooft, G. Quantum gravity as a dissipative deterministic system. Classical Quant. Grav. 1999, $16,3263-3279$.

21. 't Hooft, G. A mathematical theory for deterministic quantum mechanics. J. Phys. Conf. Series 2007, 67, 012015.

22. Blasone, M.; Jizba, P.; Vitiello, G. Dissipation and quantization. Phys. Lett. 2001, A287, 205-210.

23. Blasone, M.; Celeghini, E.; Jizba, P.; Vitiello, G. Quantization, group contraction and zero point energy. Phys. Lett. 2003, A310, 393-399.

24. Blasone, M.; Jizba, P.; Scardigli, F.; Vitiello, G. Dissipation and quantization in composite systems. Phys. Lett. 2009, A373, 4106-4112.

25. Marzuoli, A.; Raffa, F.A.; Rasetti, M. Algebraic characterization of bosons. In preparation.

26. Bunde, A.; Havlin, S. Fractals in Science; Springer-Verlag: Berlin, Germany, 1995.

27. Peitgen, H.O.; Jürgens, H.; Saupe, D. Chaos and Fractals: New Frontiers of Science; Springer-Verlag: Berlin, Germany, 1986.

28. Andronov, A.A.; Vitt, A.A.; Khaikin, S.E. Theory of Oscillators; Dover Publications, Inc.: Mineola, NY, USA, 1966.

29. Yuen, H.P. Two-photon coherent states of the radiation field. Phys. Rev. 1976, 13, 2226-2243.

30. Sivasubramanian, S.; Srivastava, Y.N.; Vitiello, G.; Widom, A. Quantum dissipation induced noncommutative geometry. Phys. Lett. 2003, A311, 97-105.

31. Celeghini, E.; De Martino, S.; De Siena, S.; Iorio, A.; Rasetti, M.; Vitiello, G. Thermo field dynamics and quantum algebras. Phys. Lett. 1998, A244, 455-461. 
32. Vitiello, G. Links. Relating different physical systems through the common QFT algebraic structure. In Quantum Analogues: From Phase Transitions to Black Holes and Cosmology; Lectures Notes in Physics 718; Unruh, W.G., Schuetzhold, R., Eds.; Springer: Berlin, Germany, 2007; pp. 165-205.

33. Iorio, A.; Vitiello, G. Quantum groups and Von Neumann theorem. Mod. Phys. Lett. 1994, B8, 269-276.

34. Vitiello, G. Struttura e Funzione. Una visione ecologica integrata. Riv. Filos. Neo-Scolast. 2012, 4, 625-637.

35. Montagnier, L.; Aissa, J.; Del Giudice, E.; Lavallee, C.; Tedeschi, A.; Vitiello, G. DNA waves and water. J. Phys. Conf. Series 2011, 306, 012007.

36. Pashaev, O.K.; Nalci, S. Golden quantum oscillator and Binet-Fibonacci calculus. J. Phys. A Math. Theor. 2012, 45, 015303.

\section{Appendix}

\section{The Golden Spiral and the Fibonacci Progression}

Let $d_{g} \equiv(\ln \phi) /(\pi / 2)$, where $\phi$ denotes the golden ratio, $\phi=(1+\sqrt{5}) / 2$, and the subscript $g$ stays for golden. The logarithmic spiral with polar equation $r_{g}(\theta)=r_{0} e^{d_{g} \theta}$ is then called the golden spiral [27]. As $\theta$ grows of $\pi / 2$, the radius of the golden spiral grows in geometrical progression of ratio $\phi: r_{g}(\theta+n \pi / 2)=r_{0} e^{d_{g}(\theta+n \pi / 2)}=r_{0} e^{d_{g} \theta} \phi^{n}$ and $r_{g, n} \equiv r_{g}(\theta=n \pi / 2)=r_{0} \phi^{n}, n=0,1,2,3, \ldots$.

An "approximate" construction of the golden spiral is obtained by drawing [27] squares whose sides are in the Fibonacci progression (Fibonacci tiling), 0,1, 1, 2, 3, 5, 8, 13, ... (the Fibonacci generic number is $F_{n}=F_{n-1}+F_{n-2}$, with $\left.F_{0}=0 ; \quad F_{1}=1\right)$. The Fibonacci spiral is obtained from quarter-circles tangent to the interior of each square. It does not perfectly overlap with the golden spiral since $F_{n} / F_{n-1} \rightarrow \phi$ in the $n \rightarrow \infty$ limit, but is not equal to $\phi$ for given finite $n$.

The golden ratio $\phi$ and its "conjugate" $\psi=1-\phi=-1 / \phi=(1-\sqrt{5}) / 2$ are solutions of the "quadratic formula":

$$
x^{2}-x-1=0
$$

and of the recurrence equation $x^{n}-x^{n-1}-x^{n-2}=0$, which, for $n=2$, is the relation (47). This is satisfied also by the geometric progression of ratio $\phi$ of the radii $r_{g, n}=r_{0} \phi^{n}$ of the golden spiral. Equation (47) is the characteristic equation of the differential equation $\ddot{r}+\dot{r}-r=0$. It admits as solution $r(t)=r_{0} e^{i \omega t} e^{+d \theta(t)}$ with $\omega= \pm i \sqrt{5} / 2$ and $\theta=-t /(2 d)+c$, with $c, r_{0}$ and $d$ constants. By setting $c=0, r(t)=r_{0} e^{\mp \sqrt{5} t / 2} e^{-t / 2}$, i.e., $r_{\phi}(t)=r_{0} e^{-\phi t}$ and $r_{\psi}(t)=r_{0} e^{-\psi t}$. See [36] for details on the relation between $q$-groups and the Fibonacci progression.

(c) 2014 by the author; licensee MDPI, Basel, Switzerland. This article is an open access article distributed under the terms and conditions of the Creative Commons Attribution license (http://creativecommons.org/licenses/by/3.0/). 